\title{
Autonomía profesional y acceso directo en fisioterapia
}

\author{
Professional autonomy and direct access in physiotherapy
}

\author{
S. Souto Camba
}

La Confederación Mundial por la Fisioterapia (WCPT), máximo organismo internacional de representación de Fisioterapia, reconoce la Fisioterapia como una profesión autónoma ${ }^{1}$.

Esto supone que los fisioterapeutas han de tener la libertad de ejercer su juicio profesional y tomar decisiones, independientemente del contexto en el que desarrollen su actividad profesional, siempre que estas estén dentro de su ámbito de conocimiento y competencia. No obstante, y pesar de este importante reconocimiento por parte de la WCPT, la realidad del alcance de esta autonomía en la práctica profesional experimenta variaciones entre los diferentes países que la integran, sujetos a sus propios marcos legislativos y reguladores.

En el caso de la región europea, constituida por las organizaciones profesionales de la fisioterapia de 37 países, existe una gran diversidad en relación con este tema, coexistiendo realidades como la de Suecia o el Reino Unido, en el que el fisioterapeuta ejerce con plena autonomía en los ámbitos público y privado, frente a otros países en los que el fisioterapeuta es considerado un técnico o auxiliar subordinado en su ejercicio profesional al criterio o juicio de terceros, normalmente pertenecientes a la profesión médica.

Es por ello que en los últimos años la cuestión de la autonomía profesional ha estado presente en los diferentes congresos y comités generales de la región europea, de tal manera que, en la actualidad, impulsar el reconocimiento de la fisioterapia como profesión autónoma constituye uno de los cuatro objetivos estratégicos de la región para los próximos años ${ }^{2}$, junto con los de promover el papel del fisioterapeuta en la promoción de la salud y la prevención de la enfermedad, estimular los nexos de unión entre la educación en fisioterapia y las necesidades futuras de la profesión e influir las políticas comunitarias y defender los intereses de la fisioterapia y sus usuarios a nivel de la Unión Europea, a través de su participación activa en diferentes organismos relacionados con la salud, como son el European Health Forum y la EU Platform for Action on Diet, Physical Activity and Health, entre otros.

Si bien el debate sobre autonomía profesional se ha unido de manera significativa al del acceso directo de los pacientes a la consulta de fisioterapia como una expresión de la misma, existen otros elementos esenciales a la hora de hablar de ejercicio autónomo de la fisioterapia. Entre ellos, y siguiendo a Fernández Cervantes ${ }^{3}$, podemos citar la capacidad de emitir diagnósticos relacionados con las alteraciones de función a nivel corporal y de la persona, la capacidad de solicitar pruebas diagnósticas básicas relacionadas con la elaboración de estos diagnósticos, la capacidad para prescribir no solo el programa de intervención terapéutico, sino también cualquier otro producto sanitario necesarios para el tratamiento de Fisioterapia, la capacidad para derivar a otros profesionales del sistema de salud o la capacidad de liderar y coordinar procesos de atención sanitaria complejos.

John $\emptyset_{\text {vretveit }}{ }^{4}$ destaca la necesidad de categorizar la autonomía profesional en diferentes tipos, de cara a identificar los aspectos particulares de la misma en Fisioterapia. Así, habla de la autonomía profesional en 3 categorías o niveles: el nacional, el local y el individual, cada uno de ellos con diferentes implicaciones a nivel organizativo.

Dentro del nivel nacional, que podríamos considerar macro, se sitúan las políticas desarrolladas por el Estado relacionadas con la salud y con la economía de la salud (a su vez determinadas por las políticas comunitarias). En el momento actual, estas políticas están muy influidas por la grave crisis financiera que atraviesa la zona euro, que unida a los cambios demográficos y el incremento de la cronicidad, puede ofrecer un contexto global propicio para la fisioterapia, que ofrece soluciones a este tipo de problemas. La evidencia disponible hasta el momento nos indica que el acceso directo a fisioterapia como modelo de prestación de servicios es seguro, efectivo y aceptable, particularmente en el tratamiento de los problemas musculoesqueléticos. Es probable que resulte en un ahorro de costes sanitarios y que la calidad de la 
atención mejore. Esta evidencia debe ser utilizada para informar a los gestores y responsables de las decisiones políticas de cara a mejorar la autonomía profesional. Existen ejemplos de buena práctica a nivel internacional que pueden y deben utilizarse a tal fin, como son el desarrollado por el grupo de trabajo de Lesley Holdsworth dentro del Sistema Nacional de Salud escocés ${ }^{5}$.

Por otro lado, se encuentran las asociaciones/organizaciones profesionales que acuerdan los estándares de práctica profesional y definen los códigos deontológicos que han de guiar el ejercicio de la profesión en cada país. Estos códigos establecen los límites en aquello que una entidad empleadora puede pedir a un miembro de estas organizaciones profesionales, protegiendo al profesional frente a demandas o instrucciones que puedan infringir los códigos y estándares de práctica. Es una importante responsabilidad de las organizaciones nacionales el hacer que estos estándares recojan y definan las competencias profesionales del fisioterapeuta, siendo ambiciosos en su formulación, permitiendo la adaptación de la profesión a los nuevos avances que se puedan producir. Además, se han de constituir como lobbies de presión para provocar aquellos cambios legislativos que se entienda pueden suponer un obstáculo a la autonomía profesional. Sirva como ejemplo el caso del Reino Unido, que en el año 2012 ha conseguido para los fisioterapeutas de ese país la capacidad de prescribir medicamentos (como puede ser los AINE o la toxina botulínica) al considerarlos complemento necesario al tratamiento de fisioterapia en determinados procesos. Esto, evidentemente, se produce acompañado del necesario cambio normativo, y del desarrollo de una formación reglada que han de realizar aquellos fisioterapeutas que quieran alcanzar dicha competencia.

Una forma de autonomía muy relacionada con lo anterior en este nivel es el derecho de una profesión a regular su propia formación y curriculum. En ese sentido, en España el proceso de desarrollo de competencias profesionales se ha vinculado al proceso de desarrollo académico, gracias a la participación de los profesionales de la Fisioterapia en el diseño y la aplicación de los planes de estudio en las titulaciones de Fisioterapia. Sin embargo, así como es posible decir que la fisioterapia española ha alcanzado la plenitud en su desarrollo académico, de manera que los fisioterapeutas pueden acceder a los niveles educativos más altos siguiendo itinerarios formativos que responden a sus intereses ${ }^{3}$, a nivel profesional aún existen importantes retos que asumir de cara a alcanzar el culmen de desarrollo expresado a través de la plena autonomía profesional.

En un nivel local, que podríamos llamar meso o intermedio, la forma de autonomía profesional ante la que nos encontramos es la autodirección, entendida como el derecho de un grupo profesional determinado a ser gestionado o dirigido por miembros de la misma ocupación. Otro aspecto de la autodirección que contribuye a la autonomía es el derecho a la representación directa. Solo un fisioterapeuta entenderá las necesidades y las características del ejercicio de la Fisioterapia, y podrá de manera adecuada aconsejar a las autoridades locales, regionales o autonómicas en salud sobre cómo la fisioterapia puede contribuir al desarrollo y optimización de los servicios en salud prestados. Esto que parece tan razonable y natural, es frecuente que no se dé en los organigramas que establecen las dependencias funcionales de los grandes centros hospitalarios de nuestro país, en los que fisioterapia aparece como dependiente de las direcciones de enfermería. Tampoco se sostiene que el control y la organización de los servicios de Fisioterapia sean realizados por médicos de otra especialidad, principalmente de rehabilitación. Por ello, es tan importante que los fisioterapeutas estén representados en los comités locales o regionales de toma de decisiones (gerencias hospitalarias, gerencias de atención primaria, sindicatos, órganos de dirección, etc.).

Finalmente, y siguiendo con la categorización propuesta por $\emptyset$ vretveit, la mayor parte de las veces que se emplea el término de autonomía profesional es para hacer referencia a fenómenos situados en un nivel individual o micro. Es en este nivel donde se sitúa el derecho de un profesional a valorar y examinar a un paciente, a rechazarlo o referirlo a otro profesional cuando lo estime necesario, a prescribir un determinado tratamiento consensuado con el paciente, el derecho a darle el alta y el derecho a tratarlo sin derivación de ningún tipo. Esta forma de autonomía expresa que el profesional ha alcanzado un grado de madurez que le permite tomar plena responsabilidad de sus decisiones clínicas. Su desarrollo tiene mucho que ver con el proceso de especialización y la existencia de profesionales clínicos más competentes, teóricamente orientados y ambiciosos en la búsqueda de un ejercicio profesional independiente, sin tutorización o prescripción por parte de terceros. Los fisioterapeutas han de estar en condiciones de realizar una práctica basada en la evidencia, considerar el coste efectividad de sus intervenciones y hacer a los usuarios del sistema de salud parte del proceso clínico de toma de decisiones, respetando la propia autonomía del paciente. Y todo ello, con el compromiso firme hacia el desarrollo profesional continuo.

En un intento de síntesis, podríamos sentar las bases de la autonomía profesional en fisioterapia sobre tres pilares: $a$ ) el derecho de primer contacto o acceso directo; $b$ ) el control y la determinación de los servicios de fisioterapia por parte de la profesión, entendiendo estos servicios de manera amplia, y $c$ ) finalmente, y a consecuencia de lo anterior, la práctica profesional sin prescripción médica.

Excede el objetivo de este editorial el analizar el grado de autonomía profesional en España. Es ampliamente conocido que en el ámbito privado los fisioterapeutas reciben directamente al usuario y 
tienen libertad de criterio a la hora de programar los tratamientos. Sin embargo, si se incorporan otras de las variables a la ecuación de la autonomía, aparecen zonas oscuras que ofrecen un panorama menos positivo, encontrando limitaciones o restricciones a la práctica autónoma en nuestro país, sobre todo, y de manera preocupante, en el sistema público de salud.

El alcance de la autonomía profesional como meta está influido por multiplicidad de factores, como son la educación - que ha de satisfacer simultáneamente los requisitos de las universidades, de los órganos reguladores de la profesión y de los empleadores-, el desarrollo de una mayor evidencia para la práctica, la propia autonomía del paciente, la relación con otras profesiones del ámbito sanitario, el marco legislativo y regulador de la profesión, los modelos de gestión y organización sanitaria, y las actitudes de los propios profesionales.

Entre todos, cabe destacar la relevancia del liderazgo profesional ejercido por las organizaciones profesionales, a nivel nacional e internacional, y de líderes de opinión o expertos, de cara a generar el pensamiento crítico necesario y diseñar las estrategias o planes explícitos, visionarios y bien dirigidos que permitan a la fisioterapia alcanzar su lugar dentro de los sistemas de salud y reivindicar la autonomía profesional.

Como disciplina, la fisioterapia ha de seguir profundizando en el desarrollo de un modelo conceptual propio, vinculado al ámbito de la discapacidad, la salud y la dependencia, alejándose del modelo médico tradicional que la considera complementaria a los tratamientos médicos.

Siguiendo las reflexiones de la catedrática de Fisioterapia de la Universidad de Brighton Ann Moore ${ }^{6}$, las cuestiones de identidad también son de gran relevancia para el reconocimiento y el desarrollo de la autonomía profesional. Tener clara la respuesta a cuestiones del tipo, cómo nos percibimos como fisioterapeutas, cómo describimos lo que hacemos y cómo lo articulamos, nos permitirá distinguirnos a nosotros mismos como profesionales plenamente responsables de nuestros actos. Será a partir de ese momento cuando podremos esperar esa misma aceptación o reconocimiento por parte de los otros (entendiendo por los otros a otras profesiones de las ciencias de la salud, los usuarios de los servicios, los gestores, etc.). No es una reflexión menor, si tenemos en cuenta que en nuestro país, y según datos del Instituto Nacional de Estadística correspondientes al año 2011, el 68,47\% de los fisioterapeutas colegiados en España tienen menos de 35 años, porcentaje que aumenta hasta el 91,19\% al poner el corte de edad en los 44 años (http://www.ine.es/jaxi/tabla.do?path=/t15/p416/a2011/10/\&file=s07003.px\&type= pcaxis $\& \mathrm{~L}=0$ ).

Ejercer con autonomía implica madurez y la asunción de un mayor número de responsabilidades y obligaciones profesionales hacia los usuarios de los servicios y en última instancia hacia la sociedad. Como profesión, siempre hemos tenido presente que lo que nos guía en este movimiento continúo y hacia delante es mejorar la salud y bienestar de los ciudadanos, cambiar la realidad para que esta sea mejor. La búsqueda de este objetivo común nos ha permitido avanzar con unidad, por encima de otro tipo de intereses partidarios o corporativos. Así ha sido hasta hoy, y espero que así siga siendo en un futuro.

\section{Bibliografía}

1. WCPT. Policy Statement: Autonomy. WCPT, London (2011).

2. S. Souto. Autonomía profesional y acceso directo en fisioterapia. Congreso Nacional de Fisioterapia, Madrid, marzo (2012).

3. Fernández R, Souto A. Proceso de adquisición y propuesta de ampliación de las competencias profesionales en Fisioterapia en España. VIII Jornadas Nacionales de Educación en Fisioterapia. Ponferrada, abril 2010.

4. J. Øvretveit. Medical dominance and the development of professional autonomy in physiotherapy. Sociology of Health \& Illness, 7 (1985), pp. 76-93.

5. L.K. Holdsworth, V.S. Webster, A.K. McFadyen. What are the costs to NHS Scotland of self-referral to physiotherapy? Results of a national trial. Physiotherapy, 93 (2007), pp. 3-11.

6. Moore A. Advancing the professional profile. Continuing professional development: The journey to autonomy. 3rd European Congress on Physiotherapy Education. Viena, noviembre de 2012 